\title{
Inflation with the Starobinsky potential in Loop Quantum Cosmology
}

\author{
Béatrice Bonga* and Brajesh Gupt ${ }^{\dagger}$ \\ Institute for Gravitation and the Cosmos $\&$ Physics Department, \\ The Pennsylvania State University, University Park, PA 16802 U.S.A.
}

\begin{abstract}
A self-consistent pre-inflationary extension of the inflationary scenario with the Starobinsky potential, favored by Planck data, is studied using techniques from loop quantum cosmology (LQC). The results are compared with the quadratic potential previously studied. Planck scale completion of the inflationary paradigm and observable signatures of LQC are found to be robust under the change of the inflaton potential. The entire evolution, from the quantum bounce all the way to the end of inflation, is compatible with observations. Occurrence of desired slow-roll phase is almost inevitable and natural initial conditions exist for both the background and perturbations for which the resulting power spectrum agrees with recent observations. There exist initial data for which the quantum gravitational corrections to the power spectrum are potentially observable.
\end{abstract}

The inflationary scenario is highly successful in explaining, with minimal assumptions, the primordial origin of structure formation and small inhomogeneities observed in the cosmic microwave background (CMB). There is a plethora of inflationary models whose predictions can be matched with observations. Recent data from Planck show that, for single field inflation, the Starobinsky potential is favored over others, such as the quadratic one [1]. The standard inflationary models are based on classical general relativity and therefore inherit the big bang singularity. This leaves several conceptual issues unaddressed which are expected to be resolved by a fundamental quantum gravity theory. For instance: (i) Is there a consistent pre-inflationary extension that admits a finite quantum gravity regime and confronts the problem of a past singularity? (ii) If so, does inflation occur naturally without the need of fine tuning the initial conditions? (iii) Do the perturbations remain in a Bunch-Davies state at the onset of inflation? (iv) How strongly do the predictions of such a fundamental theory depend on the model of inflation?

Loop quantum cosmology (LQC) provides an excellent avenue to address these issues. The underlying quantum geometric effects of LQC modify the Planck scale physics leading to the resolution of classical singularities in a variety of homogeneous and inhomogeneous cosmological settings 2, 3] (see, e.g., 4] for a review). In all these models, the big bang is replaced by a quantum bounce, evolution is deterministic and all curvature scalars remain finite throughout the evolution 11 In this Letter, we investigate the aforementioned issues for single field inflation with the Starobinsky potential in LQC using the framework of [10, 11] and compare our results with the quadratic potential studied in [13 15].

Note that a priori there is no reason to believe that the pre-inflationary dynamics of the Starobinsky poten-

\footnotetext{
${ }^{1}$ For other approaches to bouncing models see, e.g. 5-8. Also see [9] (and references therein) for a recent review.
}

tial and quadratic potential are similar because: (i) already their inflationary dynamics is quite different; and (ii) the evolution equation for the scalar perturbations depends explicitly on the potential. We find that despite these differences, not only is the occurrence of slow-roll almost inevitable as is the case for the quadratic potential, but also the observable LQC corrections to the power spectrum remain similar to those for the quadratic potential. Hence, LQC predictions are robust. Furthermore, there are interesting signatures of the quantum geometry on long wavelength modes which can modify inflationary tensor fossils and can couple with observable modes as suggested in [16, 17] leaving imprints on the primordial power spectrum. This potentially opens new avenues to explore the origin of CMB anomalies in quantum gravity.

Framework: In LQC, the background quantum geometry is described by a wavefunction which is a solution of the quantum Hamiltonian constraint. For sharply peaked wavefunctions, which are peaked at classical solutions at late times, the leading quantum corrections to the background spacetime can be captured by the effective description of LQC, given by the following modified Friedmann and Raychaudhuri equations [18 20:

$$
\begin{aligned}
H^{2} & :=\left(\frac{\dot{a}}{a}\right)^{2}=\frac{8 \pi G}{3} \rho\left(1-\frac{\rho}{\rho_{\max }}\right), \\
\dot{H} & =-4 \pi G(\rho+P)\left(1-2 \frac{\rho}{\rho_{\max }}\right),
\end{aligned}
$$

where $H$ is the Hubble rate, $a$ is the scale factor, $\rho_{\max }=$ $18 \pi / \Delta_{o}^{3} \rho_{\mathrm{Pl}} \approx 0.41 \rho_{\mathrm{Pl}}$ is the universal maximum of the energy density and $\Delta_{o} \approx 5.17$ is the minimum eigenvalue of the area operator, whose value is fixed via black hole entropy calculations in loop quantum gravity [21, 22]. The LQC evolution of spacetime is completely described by (1) and (2) which, given a matter source with the energy density $\rho$ and pressure $P$ form a well posed initial value problem. Note that the LQC modifications are dominant only in the quantum gravity regime, where the energy density of the matter field is Planckian. When 
the spacetime curvature is sub-Planckian $\left(\rho \ll \rho_{\max }\right)$, equations (1) and (2) reduce to the classical Friedmann and Raychaudhuri equations.

In the inflationary model under consideration, the matter source is a scalar field with a self-interacting potential, which also drives inflation [23 27]:

$$
V(\phi)=\frac{3 M^{2}}{32 \pi G}\left(1-e^{-\sqrt{\frac{16 \pi G}{3}} \phi}\right)^{2},
$$

where $M$ is the mass of the inflaton $\phi$ with $\rho=\dot{\phi}^{2} / 2+$ $V(\phi)$ and $P=\dot{\phi}^{2} / 2-V(\phi)$. This potential is commonly known as the Starobinsky potential and is related to various other potentials, e.g. Higgs inflation, via $\alpha$-attractors 28]. It is evident from the above expression that $V(\phi)$ increases exponentially for $\phi<0$, has a minimum at $\phi=0$ and approaches a constant value $\left(3 M^{2} / 32 \pi G\right)$ as $\phi \rightarrow \infty$. The form of the Klein-Gordon equation in LQC remains unchanged:

$$
\ddot{\phi}+3 H \dot{\phi}+\partial_{\phi} V(\phi)=0,
$$

which reflects the fact that LQC corrections are purely quantum geometrical and do not require any modification or violation of the standard energy conditions.

In the standard inflationary scenario, by using Einstein's equations and the slow-roll conditions together with data observed by the Planck mission [1, we are led to set $M=2.51 \times 10^{-6} m_{\mathrm{Pl}}$. In particular, we used the value of the amplitude of the scalar power spectrum, its running, and the constraints on the number of e-folds $\left(N_{*}\right)$ from the time the reference mode $k_{*}$ exited the horizon to the end of inflation ${ }^{2}$ This procedure also defines the phase of desired slow-roll which is required for compatibility with observations. Thus, this phase always refers to the last $N_{*}$ e-folds of inflation, but of course a phase of accelerated expansion can precede the desired slow-roll period. At the onset of the desired slow-roll, $\phi=1.08 m_{\mathrm{Pl}}$ and the first two Hubble slow-roll parameters are $\epsilon=1.98 \times 10^{-4}$ and $\delta=-1.73 \times 10^{-2}$. Inflation ends when $\epsilon=1$ which corresponds to $\phi=0.19 \mathrm{~m}_{\mathrm{Pl}}$.

To understand the evolution of quantum perturbations one needs a formalism of quantum fields on quantum cosmological spacetime, which -at first- seems an intractable task. It turns out that within the test field

\footnotetext{
2 Here, in order to compute the mass parameter, we have assumed that the LQC corrections to the power spectrum are extremely small at the pivot scale $k_{*}$. This assumption does indeed hold in the numerical results discussed here. However, in principle, this is inconsistent and a proper way to address this would require significant numerical work along the lines of [29]. There the authors find that, while this assumption is conceptually important, it leaves the main results practically unchanged for the quadratic potential; we expect the same to be true for the Starobinsky potential.
}

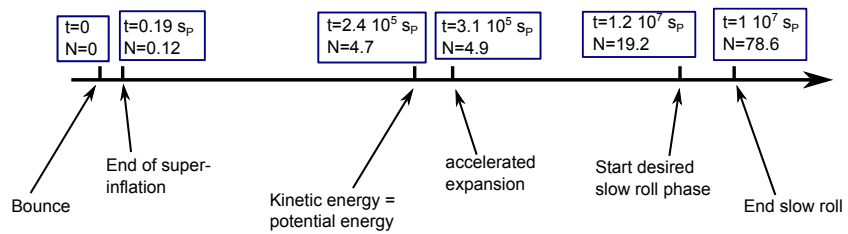

FIG. 1. Time line of evolution of the background spacetime with $\phi_{\mathrm{B}}=-1.41 m_{\mathrm{Pl}}$ and $\dot{\phi}_{\mathrm{B}}>0$.

approximation (that is, the backreaction of the perturbations on the background quantum geometry is negligible), surprising simplifications occur: The dynamics of the perturbations on the quantum FLRW geometry is completely equivalent to that of perturbations evolving on a 'dressed' FLRW metric, where 'dressing' refers to quantum corrections [10, 11] (for other approaches within LQC, see 30 35]). Interestingly, for sharply peaked states, the dressed metric is extremely well described by eq. (1) and 22] [20, 36].

Quantum bounce and slow-roll: The key feature of homogeneous and isotropic spacetimes in LQC is the occurrence of a quantum bounce when the energy density of the matter field reaches its universal maximum, i.e. $\rho=\rho_{\max }$. Here, the Hubble rate $H$ is zero (eq. (1)), $\dot{H}$ is positive (eq. 2) and therefore the scale factor has a minimum. Following the bounce, there is a phase of super-inflation during which the energy density decreases while the Hubble rate increases very rapidly and takes its maximum value at $\rho=\rho_{\max } / 2$. In further evolution, both the energy density and the Hubble rate continue to decrease monotonically. A typical time line of the post bounce evolution is shown in Fig.1. Inflation starts when the energy density of the matter field is of the order of $10^{-12} \rho_{\text {Pl }}$ where LQC effects are negligible (for a detailed analysis of the background evolution starting from the bounce till the end of inflation, see the accompanying paper [37]). Hence, LQC provides a Planck scale completion of the inflationary scenario while resolving the classical singularity. The question now is: How natural is the occurrence of inflation in this model? That is, what fraction of the initial data surface leads to the desired slow-roll.

To understand this, let us consider the space of initial conditions at the bounce which is 4 dimensional: $\left(a_{\mathrm{B}}, H_{\mathrm{B}}, \phi_{\mathrm{B}}, \dot{\phi}_{\mathrm{B}}\right)$. Utilizing the rescaling freedom of the scale factor we fix $a_{\mathrm{B}}=1$ without loss of any generality. Furthermore, since $H_{\mathrm{B}}=0$, there are only two degrees of freedom in the space of initial conditions at the bounce: $\phi_{\mathrm{B}}$ and $\dot{\phi}_{\mathrm{B}}$. The energy density at the bounce is bounded by $\rho_{\max }$ which yields: $\rho_{\max }=\dot{\phi}_{\mathrm{B}}{ }^{2} / 2+V\left(\phi_{\mathrm{B}}\right)$. Hence, the entire space of initial conditions at the bounce is captured by $\phi_{\mathrm{B}}$ and $\dot{\phi}_{\mathrm{B}}$, whose range is given by: $\phi_{\mathrm{B}} \in\left[-3.47 m_{\mathrm{Pl}}, \infty\right)$ and $\left|\dot{\phi}_{\mathrm{B}}\right| \leq 0.905 m_{\mathrm{Pl}}^{2}$. Now the question is: What fraction of this space of initial condi- 
tions give the desired slow-roll phase in the future evolution? It turns out that the observationally compatible initial conditions leading to the desired slow-roll phase are: $\phi_{\mathrm{B}} \geq-1.45 m_{\mathrm{Pl}}$ for $\dot{\phi}_{\mathrm{B}}>0$ and $\phi_{\mathrm{B}} \geq 3.63 m_{\mathrm{Pl}}$ for $\dot{\phi}_{\mathrm{B}}<0$. The remaining initial conditions either do not give inflation at all or do not have a sufficient number of e-folds required for compatibility with observations. Unlike the quadratic potential, the space of initial conditions - defined by the constant energy density surface at the bounce- is unbounded, because the plateau-side of the potential continues all the way to infinity and is well below $\rho_{\max }$. Therefore, for a uniform measure the volume of the whole space of initial conditions for the Starobinsky potential is infinite and it is difficult to quantify the likelihood of inflation, as was done for the quadratic potential in $12,14,38,3$ However, since only a tiny fraction of initial conditions do not lead to inflation it seems that, qualitatively, most of the initial conditions do lead to the desired slow-roll phase.

Interestingly, all of these initial conditions are kinetic energy dominated at the bounce. The small fraction of initial data that does not lead to the desired slowroll phase are either potential dominated or do not have enough initial kinetic energy. This is in striking contrast with the quadratic potential, where in fact all potential energy dominated bounces lead to the desired slow-roll phase and have a huge number of e-folds from the bounce till the end of inflation. However, it is the kinetic energy dominated initial conditions for which LQC corrections to the primordial power spectrum are in the observable range for the quadratic potential. For the kinetic energy dominated initial conditions the inflaton behaves essentially as a massless scalar field near the bounce and consequently, the details of the potential are irrelevant there. Therefore, while the observational consequences of the inflationary dynamics for the two potentials are quite different, the pre-inflationary dynamics is very similar.

Let us now consider the evolution of quantum perturbations, described by the Fourier transform of the gauge invariant Mukhanov-Sasaki scalar mode $q_{k}$ and two tensor modes $e_{k}$, on the quantum geometry.

Primordial power spectrum: The evolution equation for $q_{k}$ and $e_{k}$, where $k$ is the comoving wavenumber, propagating on the quantum modified dressed geometry is given by [11, 15]:

\footnotetext{
3 This issue could be resolved for potentials, which are related to Starobinsky potential via $\alpha$-attractors, that have a plateau for a finite range in $\phi$. For such potentials the space of initial conditions at the bounce will be compact and a regular measure suffices to talk about probabilities. For instance, the Higgs potential satisfies this criterion as it has a plateau region in the center and exponential walls on both sides.
}

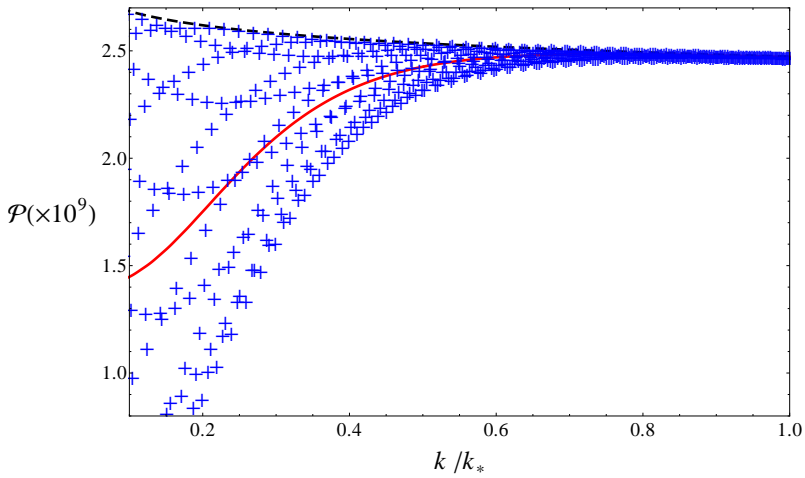

FIG. 2. Scalar power spectrum for Starobinsky potential with $\phi_{\mathrm{B}}=-1.42 m_{\mathrm{Pl}}$ and $\dot{\phi}_{\mathrm{B}}>0$. The blue ' + ' points show $\mathcal{P}_{\mathrm{LQC}}$ which rapidly oscillates with $k$. The average is shown by (red) solid curve, and $\mathcal{P}_{\mathrm{BD}}$ is shown by (black) dashed curve.

$$
\begin{aligned}
& q_{k}^{\prime \prime}(\tilde{\eta})+2 \frac{\tilde{a}^{\prime}}{\tilde{a}} q_{k}^{\prime}(\tilde{\eta})+\left(k^{2}+\tilde{\mathcal{U}}(\tilde{\eta})\right) q_{k}(\tilde{\eta})=0 \\
& e_{k}^{\prime \prime}(\tilde{\eta})+2 \frac{\tilde{a}^{\prime}}{\tilde{a}} e_{k}^{\prime}(\tilde{\eta})+k^{2} e_{k}(\tilde{\eta})=0
\end{aligned}
$$

where the prime denotes the derivative with respect to the dressed conformal time $\tilde{\eta}$ and $\tilde{\mathcal{U}}=$ $a^{2}\left(6 \pi G \frac{\dot{\phi}^{2}}{\rho} V(\phi)-2 \sqrt{\frac{6 \pi G \dot{\phi}^{2}}{\rho}} \frac{\partial V}{\partial \phi}+\frac{\partial^{2} V}{\partial \phi^{2}}\right)$ is the dressed effective scalar potential [11, 15].

Recall that in the standard inflationary scenario the initial conditions for the quantum perturbations are given at the onset of inflation where the spacetime metric can be approximated by a de Sitter metric. Therefore, the modes of the perturbations are taken to be in the BunchDavies vacuum. In LQC, on the other hand, since the pre-inflationary dynamics extends to the deep Planck regime where the spacetime metric cannot be approximated by a de Sitter metric, it is not meaningful to require that the modes be in the Bunch-Davies vacuum at the bounce. As described in [11, 15], we choose quantum perturbations to be in a 4th order adiabatic 'vacuum' which has the following two main properties: (i) respects the symmetry of the background spacetime and (ii) the expectation value of the renormalized energy density of the perturbations is negligible with respect to the background energy density at the bounce. Unlike in the de Sitter spacetime, this procedure does not single out a unique state.

A natural question then is: Among the admissible states at the bounce, can one choose one that would lead to a power spectrum at the end of inflation that not only agrees with the standard power spectrum at small angular scales but also leads to a power suppression at large angular scales, say, $\ell<30$ ? Interestingly, the answer is in the affirmative [39]! This means that, when the state is evolved to the onset of slow roll, the $\ell<30$ modes are not 
in the Bunch-Davies vacuum at the onset of inflation 4 The scalar power spectrum for such a vacuum state at the end of inflation is shown in Fig.2 for initial background conditions $\phi_{\mathrm{B}}=-1.42 m_{\mathrm{Pl}}$ and $\phi_{\mathrm{B}}>0$. The deviation between LQC and standard power spectrum can be understood by writing the LQC mode functions of the quantum perturbations at the onset of inflation as a Bogoliubov transformation on the Bunch-Davies states: $q(k)=$ $\alpha(k) q_{\mathrm{BD}}(k)+\beta^{*}(k) q_{\mathrm{BD}}(k)$. The LQC power spectrum can then be written as $\mathcal{P}_{\mathrm{LQC}}=\left(1+2|\beta(k)|^{2}\right) \mathcal{P}_{\mathrm{BD}}$, where $|\beta(k)|^{2}$ has the physical interpretation of the number density of the particles produced by the pre-inflationary dynamics with respect to the standard Bunch-Davies vacuum. For all choices of vacuum states, only modes with small $k$ deviate from the standard Bunch-Davies power spectrum, while for large $k$ there is remarkable agreement with the standard Bunch-Davies power spectrum. This is because for small $k$ the particle number density is non-zero $(|\beta(k)|>0)$ and $|\beta(k)|$ rapidly decays to zero for large $k$, as these modes are too energetic to be affected by background curvature and consequently do not get excited.

This qualitative feature is also true for the quadratic potential and can be understood by comparing the relevant scales. During the evolution through the quantum gravity regime the quantum perturbations can interact with the curvature and become excited. Modes whose physical wavelength is smaller than the characteristic curvature length scale $\lambda_{\mathrm{LQC}}:=\sqrt{24 \pi^{2} / \mathcal{R}_{\mathrm{B}}}$, where $\mathcal{R}_{\mathrm{B}}$ is the Ricci scalar at the bounce, propagate as if they are in flat space. Modes with physical wavelength larger than $\lambda_{\mathrm{LQC}}$ are affected by the background curvature: particle creation occurs and these modes become excited. Therefore, they no longer are in the Bunch-Davies vacuum state at the onset of inflation. Consequently, the primordial power spectrum for modes with small $k$ shows deviation from the power spectrum obtained in the standard inflationary scenario, where one assumes all modes to be in the Bunch-Davies state at the onset of inflation. Hence, the origin of the non-Bunch Davies state is a direct consequence of the pre-inflationary $L Q C$ dynamics and occur always for small $k$ regardless of the details of the inflationary model. However, the quantitative details of the power spectrum do depend on the type of potential and the nature of the bounce. For instance, if the bounce is kinetic energy dominated, the scalar field behaves like a massless scalar field in the quantum gravity regime. In such cases, the LQC corrections to the power spectrum

\footnotetext{
${ }^{4}$ Here, we only show existence of at least one state that leads to power suppression. It should be noted that there also exist states that show power enhancement for $\ell<30$. As of now, these states are at the same footing as the one chosen here that shows power suppression. The physical criteria to select states resulting in power suppression and the issue of their uniqueness are currently being investigated 39 .
}

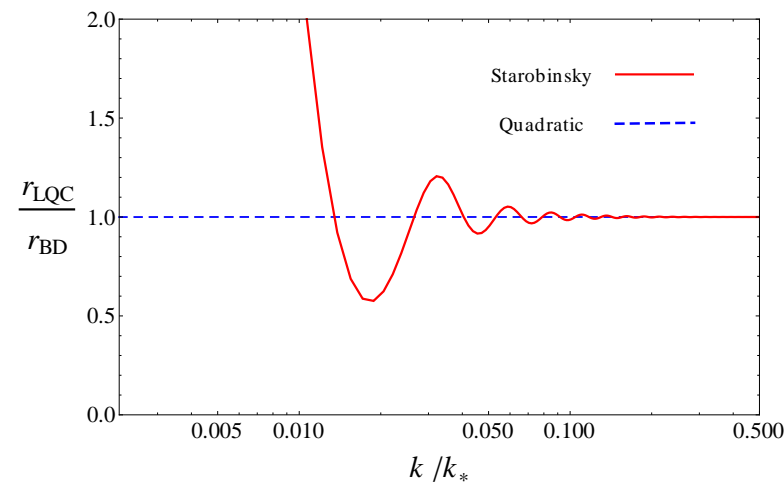

FIG. 3. Comparison of LQC corrections to tensor-to-scalar ratio between the Starobinsky and quadratic potential. States are chosen to give the maximum power suppression as in Fig.2

will be similar for all potentials. On the other hand, if the bounce is not kinetic energy dominated, then there could be potential specific signatures on the LQC corrections to the power spectrum. Thus, the LQC corrections to the power spectrum are robust under the change of potential for all kinetic energy dominated bounces including the phenomenologically interesting initial conditions for the Starobinsky potential considered here and the quadratic potential.

Imprints on long wavelength modes: So far in this Letter, we have seen that the LQC corrections to the observable power-spectrum remain robust under the change of potential. Nonetheless, there are interesting signatures of LQC pre-inflationary dynamics on super horizon modes for Starobinsky potential.

From eq. (5) and (6) it is immediately obvious that, just as in the standard inflationary paradigm, evolution of the scalar and tensor modes is different due to the presence of an effective potential $\tilde{\mathcal{U}}$ for the scalar evolution equation. Therefore, the particle density for scalar and tensor modes should be different from each other for $k^{2} \lesssim \tilde{\mathcal{U}}$. It turns out that the numerical value of $\tilde{\mathcal{U}}$ is typically very small compared to even the smallest observable wavenumber $k_{\min }$ and consequently negligible. However, a small subset of the initial data surface $\left(-1.45 m_{\mathrm{Pl}} \lesssim \phi_{\mathrm{B}} \lesssim-1.38 m_{\mathrm{Pl}}\right)$ exists for which $\tilde{\mathcal{U}}$ is of the order of $10^{-5} k_{\min }^{2}$ so that for $k \lesssim k_{\min } / 300$, the effect of $\tilde{\mathcal{U}}$ on the scalar modes is no longer negligible. The tensor and scalar particle densities are therefore different for these modes and $r_{\mathrm{LQC}} \neq r_{\mathrm{BD}}$, where $r$ is the tensorto-scalar ratio and the subscript 'BD' refers to the predictions from the standard inflationary paradigm. This is distinct from the quadratic potential, where this difference is negligible for all $k \gtrsim 10^{-5} k_{\min }$. This is shown in Fig. 3, where $r_{\mathrm{LQC}} / r_{\mathrm{BD}}$ is plotted for the Starobinsky and the quadratic potential.

The quantum gravity induced deviations from $r_{\mathrm{BD}}$ are 
not directly relevant for observational modes, but the altered behavior of these super horizon modes can change the observed three-point functions (and higher order correlation functions) through mode-mode coupling as well as play an important role for tensor fossils. This difference between the scalar and tensor modes as compared to the standard picture, could thus lead to signatures in the non-Gaussian modulation of the power spectrum due to super horizon modes [16, 17. It is noteworthy that the set of initial conditions for these effects is very small. Nonetheless, it is interesting as these initial conditions fall nicely into the regime for which the LQC corrections to the power spectrum are in the observable window. Thus, if these LQC corrections are observed, there is also hope to observe this effect. Furthermore, these initial conditions fall on the exponential side of the potential and not on the plateau side. Therefore, they are very likely to be present for potentials which have exponential walls such as the Higgs potential.

In summary, we have studied a quantum gravitational extension of the inflationary scenario with the Starobinsky potential using the framework of quantum fields on quantum cosmological spacetime in LQC. Since the preinflationary dynamics of LQC is very different from that of classical GR, it is not obvious whether inflation is even obtained if one starts in the deep Planck regime. If there is inflation, does it agree with observations? Are there any deviations from the standard power spectrum? We found that the pre-inflationary dynamics of LQC fits very well with inflation and there are natural initial conditions for both the background spacetime and perturbations that lead to the desired slow-roll phase compatible with observations. Almost all initial conditions starting at the bounce lead to the desired slow-roll phase. The LQC pre-inflationary excites the modes of the perturbations, as a result they carry excitations over the Bunch-Davies state at the onset of inflation, giving corrections to the standard inflationary power spectrum. There exist initial conditions for which these LQC induced corrections to the standard inflationary predictions at large angular scales are observable while being in complete agreement with observations at small angular scales.

Since the observationally relevant initial conditions are all kinetic energy dominated in the quantum gravity regime, the inflaton behaves essentially as a massless scalar field and the details of the potential do not affect the quantum gravity induced corrections to the observable power spectrum. Hence, the occurrence of desired inflationary phase and corrections to the primordial power spectrum are robust features of $L Q C$. Thus, LQC has matured enough to confront recent observational data while providing a new quantum gravity window to understand various CMB anomalies. A detailed analysis of the results and extensive phenomenological investigations of the model is presented in 37.

Finally, the model considered here, i.e. a minimally coupled scalar with the Starobinsky potential is on-shell conformally related to a higher derivative gravitational theory whose Lagrangian density is $R+R^{2} / 6 M^{2}$ [2327. Here we studied the pre-inflationary dynamics of the scalar field with a standard Einstein-Hilbert action where LQC quantization is well understood. An LQC treatment of the conformally related theory requires a careful study of loop quantization of higher derivative theories, which is currently being pursued [40].

Acknowledgements: We are grateful to Abhay Ashtekar for his constant guidance, extensive discussions and feedback at various stages of the preparation of this manuscript, and to Ivan Agullo for extensive discussions. We would also like to thank Aurelien Barrau and Parampreet Singh for fruitful discussions as well as the anonymous referees for their insightful comments which led to improvement in the manuscript. This work was supported by NSF grant PHY-1505411, the Eberly research funds of Penn State and a Frymoyer Fellowship to BB.

* bpb165@psu.edu

$\dagger$ bgupt@gravity.psu.edu

[1] Planck Collaboration, P. Ade et al., "Planck 2015. XX. Constraints on inflation", arXiv:1502.02114.

[2] A. Ashtekar, T. Pawlowski and P. Singh, Phys. Rev. Lett. 96, 141301 (2006) doi:10.1103/PhysRevLett.96.141301 gr-qc/0602086.

[3] A. Ashtekar, T. Pawlowski and P. Singh, Phys. Rev. D 74, 084003 (2006) doi:10.1103/PhysRevD.74.084003 grqc/0607039.

[4] A. Ashtekar and P. Singh, "Loop Quantum Cosmology: A Status Report", Class.Quant.Grav. 28 (2011) 213001, arXiv:1108.0893.

[5] J. Khoury, B. A. Ovrut, P. J. Steinhardt, and N. Turok, "The Ekpyrotic universe: Colliding branes and the origin of the hot big bang", Phys.Rev. D64 (2001) 123522, arXiv:hep-th/0103239.

[6] M. Gasperini and G. Veneziano, "The Pre-big bang scenario in string cosmology", Phys.Rept. 373 (2003) 1-212, arXiv:hep-th/0207130.

[7] J. Khoury, B. A. Ovrut, N. Seiberg, P. J. Steinhardt, and N. Turok, "From big crunch to big bang", Phys. Rev. D65 (2002) 086007, |arXiv:hep-th/0108187.

[8] R. Kallosh, L. Kofman, A. D. Linde, and A. A. Tseytlin, "BPS branes in cosmology", Phys. Rev. D64 (2001) 123524, arXiv:hep-th/0106241

[9] D. Battefeld and P. Peter, "A Critical Review of Classical Bouncing Cosmologies", Phys. Rept. 571 (2015) 1-66, arXiv: 1406.2790

[10] A. Ashtekar, W. Kaminski, and J. Lewandowski, "Quantum field theory on a cosmological, quantum space-time", Phys.Rev. D79 (2009) 064030, arXiv:0901.0933.

[11] I. Agullo, A. Ashtekar, and W. Nelson, "Extension of the quantum theory of cosmological perturbations to the Planck era", Phys.Rev. D87 (2013), no. 4, 043507, arXiv:1211.1354

[12] A. Ashtekar and D. Sloan, Phys. Lett. B 694, 108 (2011) doi:10.1016/j.physletb.2010.09.058 arXiv:0912.4093 [gr$\mathrm{qc}]$. 
[13] A. Ashtekar and D. Sloan, "Probability of Inflation in Loop Quantum Cosmology", Gen.Rel.Grav. 43 (2011) 3619-3655, arXiv:1103.2475

[14] A. Corichi and A. Karami, Phys. Rev. D 83, $104006 \quad$ (2011) doi:10.1103/PhysRevD.83.104006 arXiv:1011.4249 [gr-qc]].

[15] I. Agullo, A. Ashtekar, and W. Nelson, "The pre-inflationary dynamics of loop quantum cosmology: Confronting quantum gravity with observations", Class.Quant.Grav. 30 (2013) 085014, arXiv:1302.0254.

[16] F. Schmidt and L. Hui, "Cosmic Microwave Background Power Asymmetry from Non-Gaussian Modulation", Phys. Rev. Lett. 110 (2013) 011301, arXiv:1210.2965, [Erratum: Phys. Rev. Lett.110,059902(2013)].

[17] I. Agullo, "Loop quantum cosmology, non-Gaussianity, and CMB power asymmetry", arXiv:1507.04703

[18] V. Taveras, "Corrections to the Friedmann Equations from LQG for a Universe with a Free Scalar Field", Phys.Rev. D78 (2008) 064072, arXiv:0807.3325

[19] P. Singh, "Are loop quantum cosmos never singular?", Class.Quant.Grav. 26 (2009) 125005, arXiv:0901.2750.

[20] A. Ashtekar and B. Gupt, "Generalized effective description of loop quantum cosmology", Phys. Rev. D92 (2015), no. 8, 084060, arXiv:1509.08899.

[21] K. A. Meissner, "Black hole entropy in loop quantum gravity", Class. Quant. Grav. 21 (2004) 5245-5252, || arXiv:gr-qc/0407052

[22] M. Domagala and J. Lewandowski, "Black hole entropy from quantum geometry", Class. Quant. Grav. 21 (2004) 5233-5244, arXiv:gr-qc/0407051.

[23] J. D. Barrow, "The Premature Recollapse Problem in Closed Inflationary Universes", Nucl. Phys. B296 (1988) 697-709.

[24] J. D. Barrow and S. Cotsakis, "Inflation and the Conformal Structure of Higher Order Gravity Theories," Phys. Lett. B 214, 515 (1988).

[25] K. i. Maeda, "Towards the Einstein-Hilbert Action via Conformal Transformation," Phys. Rev. D 39, 3159 (1989). doi:10.1103/PhysRevD.39.3159

[26] A. A. Starobinsky, S. Tsujikawa, and J. Yokoyama, "Cosmological perturbations from multifield inflation in generalized Einstein theories", Nucl. Phys. B610 (2001) 383-410, arXiv:astro-ph/0107555.

[27] A. De Felice and S. Tsujikawa, "f(R) theories," Living Rev. Rel. 13, 3 (2010) doi:10.12942/lrr-2010-3 arXiv:1002.4928 [gr-qc]].
[28] M. Galante, R. Kallosh, A. Linde, and D. Roest, "Unity of Cosmological Inflation Attractors", Phys. Rev. Lett. 114 (2015), no. 14, 141302, arXiv:1412.3797.

[29] I. Agullo and N. A. Morris, "Detailed analysis of the predictions of loop quantum cosmology for the primordial power spectra," Phys. Rev. D 92, no. 12, 124040 (2015) doi:10.1103/PhysRevD.92.124040 arXiv:1509.05693 [gr$\mathrm{qc}]$.

[30] M. Fernandez-Mendez, G. A. Mena Marugan, and J. Olmedo, "Hybrid quantization of an inflationary universe", Phys. Rev. D86 (2012) 024003, arXiv:1205.1917.

[31] L. C. Gomar, M. Martn-Benito, and G. A. M. Marugn, "Gauge-Invariant Perturbations in Hybrid Quantum Cosmology", JCAP 1506 (2015), no. 06, 045, | arXiv: 1503.03907

[32] A. Barrau, T. Cailleteau, J. Grain, and J. Mielczarek, "Observational issues in loop quantum cosmology", Class. Quant. Grav. 31 (2014) 053001, arXiv:1309.6896.

[33] T. Cailleteau, J. Mielczarek, A. Barrau, and J. Grain, "Anomaly-free scalar perturbations with holonomy corrections in loop quantum cosmology", Class. Quant. Grav. 29 (2012) 095010, arXiv:1111.3535.

[34] A. Barrau, M. Bojowald, G. Calcagni, J. Grain, and M. Kagan, "Anomaly-free cosmological perturbations in effective canonical quantum gravity", JCAP $\mathbf{1 5 0 5}$ (2015), no. 05, 051, arXiv:1404.1018.

[35] M. Bojowald and G. M. Paily, "Deformed General Relativity and Effective Actions from Loop Quantum Gravity", Phys. Rev. D86 (2012) 104018, arXiv:1112.1899.

[36] I. Agullo, A. Ashtekar, and B. Gupt, "LQC phenomenology from non-semiclassical quantum states", In preparation.

[37] B. Bonga and B. Gupt, "Phenomenological investigation of a quantum gravity extension of inflation with the Starobinsky potential," Phys. Rev. D 93, no. 6, 063513 (2016) doi:10.1103/PhysRevD.93.063513 arXiv:1510.04896 [gr-qc]].

[38] A. Corichi and D. Sloan, "Inflationary Attractors and their Measures," Class. Quant. Grav. 31, 062001 (2014) doi:10.1088/0264-9381/31/6/062001 arXiv:1310.6399 [gr-qc]].

[39] A. Ashtekar and B. Gupt, In preparation, 2015.

[40] X. Zhang and Y. Ma, "Extension of loop quantum gravity to $f(R)$ theories", Phys. Rev. Lett. 106 (2011) 171301, arXiv:1101.1752 